\title{
Haddad Syndrome: A Case Report in a Filipino Infant
}

\author{
April Grace Dion-Berboso ${ }^{1}$ and Maria Melanie Liberty B. Alcausin ${ }^{1,2}$ \\ ${ }^{1}$ Department of Pediatrics, College of Medicine and Philippine General Hospital, University of the Philippines Manila \\ ${ }^{2}$ Institute of Human Genetics, National Institutes of Health, University of the Philippines Manila
}

\begin{abstract}
Haddad syndrome is a rare congenital disorder in which congenital central hypoventilation syndrome (CCHS) occurs with Hirschsprung's disease. It is extremely rare with only more than 60 cases reported in the worldwide literature. We report on a Filipino newborn male infant who presented with signs and symptoms of progressive abdominal enlargement, bowel obstruction, and recurrent hypoventilation. The diagnosis of Haddad syndrome was made clinically and confirmed by sequence analysis of the PHOX2B gene which showed a 27repeat heterozygous expansion of the polyalanine-coding region. All CCHS patients require assisted ventilation especially during sleep to prevent lung atelectasis and other complications. An early diagnosis and confirmation by genetic testing is vital for proper management of affected patients.
\end{abstract}

Key Words: Haddad syndrome, congenital central hypoventilation syndrome, Hirschsprung's disease, PHOX2B gene mutation, Filipino

\section{Introduction}

Congenital central hypoventilation syndrome (CCHS) is a rare genetic disorder with a worldwide estimate of nearly 1000 affected individuals. Fifteen to $20 \%$ of patients with CCHS also have Hirschsprung's disease, an extremely rare combination that occurs in less than 1 in 1,000,000 live births and is known as Haddad syndrome. ${ }^{1}$ There are only more than 60 cases reported in the literature. The co-occurrence of CCHS and HD suggests a common etiology involving failure of neural crest development, making HD the most common neurocristopathy associated with CCHS. ${ }^{2}$

Children with CCHS experience progressive hypercapnia and hypoxemia especially during sleep. Treatment involves ensuring adequate ventilation of patients who are unable to achieve adequate gas exchange during spontaneous breathing. ${ }^{3}$ Positive pressure ventilation via tracheostomy is recommended during the first years of life. Home mechanical ventilation can be provided through the

Poster Presented at the 13th International Congress of Human Genetics (ICHG 2016), April 2016, Kyoto, Japan.

Corresponding author: April Grace Dion-Berboso, MD

Department of Pediatrics

Philippine General Hospital

University of the Philippines Manila

Taft Avenue, Ermita, Manila 1000 Philippines

Telephone: +(63) 917-3259945

Email: adberboso@up.edu.ph use of a portable positive pressure ventilator via tracheostomy. An early diagnosis is necessary to avoid the harmful effects of hypoxemia, hypercapnia and acidosis on the neurocognitive and cardiovascular functions of affected individuals. $^{4}$

In this report, we share a case of Haddad syndrome in a Filipino infant, to elucidate its signs and symptoms, and to highlight the importance of having a high index of clinical suspicion and subsequent confirmation of the diagnosis through genetic testing in providing optimal care to affected patients.

\section{Case Report}

J. D. is a newborn male and the only child of a nonconsanguineous Filipino couple. He was born to a 28-yearold father and a 28-year-old primigravid mother who delivered preterm at 32-34 weeks age of gestation and birth weight of 2300 grams via caesarian section due to polyhydramnios. At birth, he had good cry and activity but presented with apneic episodes at 1 hour of life prompting intubation and mechanical lung ventilation. At 2 days of life, J.D. was noted to have progressive abdominal distention and absence of bowel movement which led to the clinical suspicion of Hirschprung's disease. He underwent open rectal biopsy and transverse loop colostomy at the Philippine General Hospital. Frozen section biopsy of the rectum showed absence of ganglion cells confirming the diagnosis of Hirschsprung's disease.

During J.D.'s first 2 months of stay in the hospital, recurrent episodes of shallow breathing progressing to apnea upon weaning of the mechanical ventilator respiratory rate were noted. Several attempts to extubate him failed despite clearance of his nosocomial pneumonia. On continuous positive airway pressure at $\mathrm{FiO}_{2}$ 30\% and positive end expiratory pressure (PEEP) of $5 \mathrm{cmH}_{2} \mathrm{O}$, the arterial blood gas (ABG) ( $\mathrm{pH}$ of 7.230, $\mathrm{paCO}_{2}$ of $96 \mathrm{mmHg}$, $\mathrm{pO}_{2} \mathrm{mmHg}$, of $59.5, \mathrm{HCO}_{3}$ of $40 \mathrm{mmol} / \mathrm{L}$, base excess of 9.4 $\mathrm{mmol} / \mathrm{L}$ and $\mathrm{paO}_{2}$ of $84.5 \%$ ) showed uncompensated respiratory acidosis and hypoxemia. Thirty minutes after shifting back to mechanical ventilation at $\mathrm{FiO} 230 \%$, peak inspiratory pressure (PIP) of $15 \mathrm{cmH}_{2} \mathrm{O}$, PEEP of $5 \mathrm{cmH}_{2} \mathrm{O}$ and respiratory rate of 15 , the $\mathrm{ABG}\left(\mathrm{pH}\right.$ of $7.431, \mathrm{paCO}_{2}$ of $58.1 \mathrm{mmHg}, \mathrm{pO}_{2}$ of $74.5 \mathrm{mmHg}, \mathrm{HCO}_{3}$ of $40 \mathrm{mmol} / \mathrm{L}$, base excess of $12.8 \mathrm{mmol} / \mathrm{L}$ and $\mathrm{paO}_{2}$ of $98.3 \%$ ) improved significantly showing compensated respiratory acidosis with normal oxygenation. 


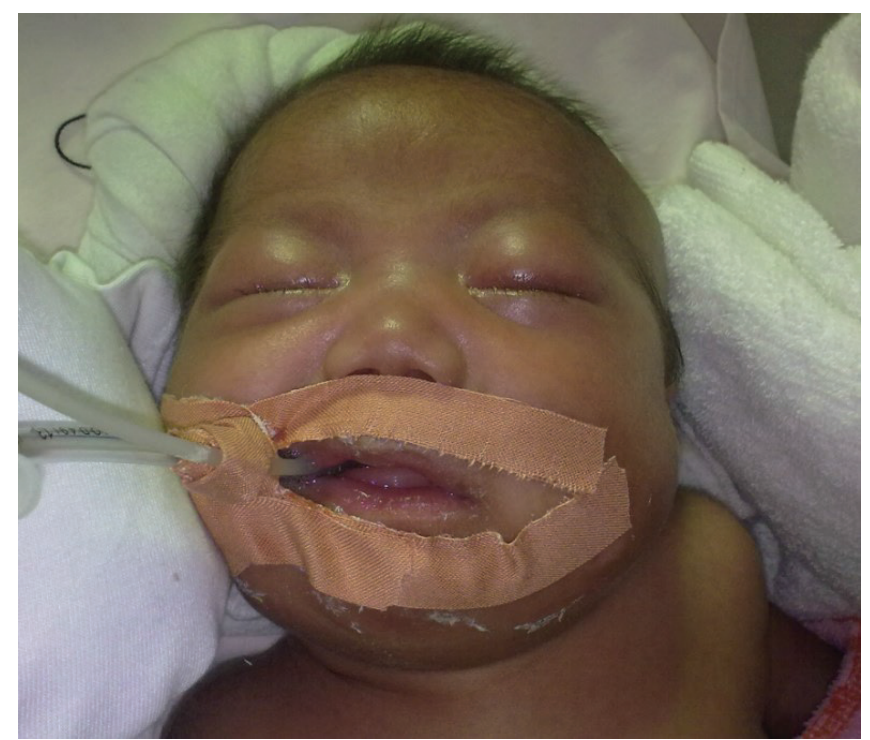

Figure 1. J.D. had puffy eyelids and a box-shaped face.

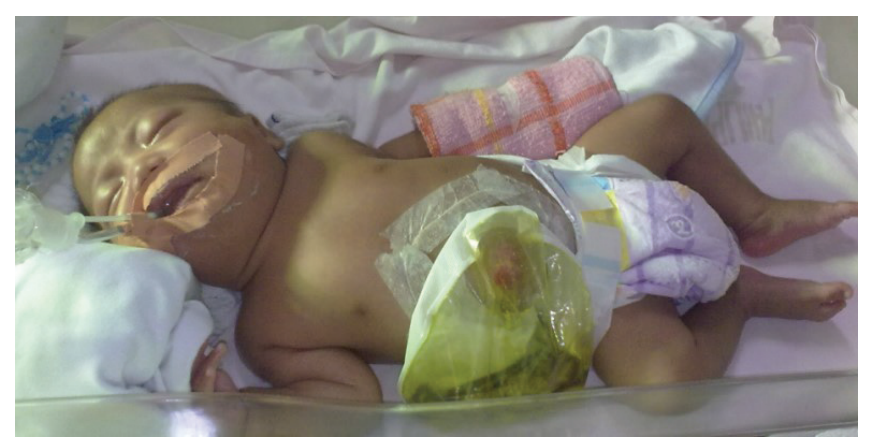

Figure 2. The patient underwent transverse loop colostomy. He was on respiratory support via an endotracheal tube.

Physical examination showed J.D. on continuous respiratory support through an endotracheal tube, with puffy eyelids, a box-shaped face, and an intact colostomy stump (Figures 1 and 2). Presumptive diagnosis of Haddad syndrome was based on the recurrent apneic episodes upon weaning from ventilator support and presence of Hirschsprung's disease. PHOX2B sequence analysis was requested to confirm the diagnosis. Pre-test genetic counseling was done and consent was sought. Sequence analysis of the PHOX2B gene done at the Children's Memorial Hospital, Chicago, Illinois, USA showed one normal PHOX2B gene coding for 20 consecutive alanines and one expanded PHOX2B gene with 27 alanine repeats. The presence of an expansion mutation confirmed the clinical diagnosis of Haddad syndrome. During the post-test genetic counseling, we advised the parents that parental testing is necessary to determine if the mutation is segregating in the family or is a novel mutation. They consented to a tracheostomy tube placement for continuous respiratory support after learning of the confirmation of the diagnosis. A few days after tracheostomy placement, J.D. was stable and was being prepared to go home on a mechanical ventilator. Unfortunately, he had another bout of severe nosocomial pneumonia which lead to respiratory failure and J.D.'s subsequent demise.

Appropriate informed consent for J.D.'s photographs to be used for medical publication was obtained.

\section{Discussion}

The essential role of PHOX2B gene in the autonomous ventilation system in humans was elucidated in a study showing heterozygous de novo mutations in PHOX2B in 18 out of 29 individuals with CCHS. Most mutations consisted of 5-9 alanine expansions within a 20-residue polyalanine tract. ${ }^{5}$ More than $90 \%$ of CCHS cases are heterozygous for an in-frame polyalanine repeat mutation (PARM) coding for 24 to 33 alanines in the mutated protein producing genotypes $20 / 24$ to $20 / 33$. The normal genotype is referred to as $20 / 20$. The remaining $10 \%$ of classical CCHS cases are heterozygous for non-polyalanine repeat mutations (NPARM) which include missense, nonsense and frameshift mutations in the PHOX2B gene. The 20/25, 20/26, and 20/27 genotypes are the most common. ${ }^{6}$

The PHOX2B gene is mapped in chromosome 4p12 and encodes a highly conserved homeobox transcription factor of 314 amino acids with two short and stable polyalanine repeats of 9 and 20 residues. ${ }^{5}$ This transcription factor is important for normal autonomic nervous system development. Mutations on this gene predisposes to a range of autonomic aberrations and loss of ventilatory drive during sleep, resulting in reduced $\mathrm{CO} 2$ and $\mathrm{O} 2$ sensitivity. The PHOX2B mutations mostly occur de novo during gametogenesis although in some cases there is a possibility of inheritance from parents with somatic mosaicism or constitutive mutation. The disease has an autosomal dominant mode of inheritance. ${ }^{4}$

Genotype phenotype studies show that individuals with the 20/25 genotype rarely require 24-hour per day ventilator support, individuals with the 20/26 genotype have variable awake needs depending upon the level of activity, and individuals with genotypes from $20 / 27$ to $20 / 33$, such as J.D., typically require continuous ventilator support. ${ }^{6}$ It is also noted in the literature that patients such as J.D. with both CCHS and HD or Haddad Syndrome are not found to have a +5 alanine expansion, but present at least a +6 alanine expansion or NPARMs. This observation agrees with the finding that short polyalanine expansions lie at the milder end of the phenotypic spectrum of a disease when compared with other types of mutations and tend to get more severe with longer alanine expansions.?

Reports show that the faces of subjects with CCHS were generally shorter and flatter resulting in the characteristic box-shaped face and an inferior inflection of the lateral $1 / 3$ of the upper vermillion border (lip trait). ${ }^{6}$ The face of J.D. had this characteristic box-like appearance (Figure 1). This 
happens because mutations in the PHOX2B gene are also expressed in the dorsal rhombencephalon, a region that gives rise to facial structures. ${ }^{4}$

CCHS is diagnosed based on the documentation of hypoventilation during sleep in the absence of primary neuromuscular, lung, cardiac or metabolic disease, or an identifiable brainstem lesion. In general, patients with CCHS have end-tidal pCO2 readings persistently above 60 torr while asleep). ${ }^{3}$

All CCHS patients require assisted ventilation during sleep. Optimal ventilation prevents atelectasis and the development of lung and cardiac complications. Portable positive pressure ventilation via tracheostomy is the most common method of providing home mechanical ventilation. ${ }^{3}$ Respiratory management is now transitioning towards noninvasive type of ventilation. Diaphragmatic pacing of the phrenic nerve of the diaphragm muscle is shown to improve ventilation and eliminate the need for continuous positive pressure ventilation support. ${ }^{8}$

In Thailand, Preutthipan et al reported that a bi-level positive airway pressure work as an invasive home ventilator via tracheostomy in two girls with congenital central hypoventilation syndrome without any complications. ${ }^{9}$ Noninvasive intermittent positive pressure ventilation delivered via a face mask using bi-level positive airway pressure has also been used. However, it is not recommended to use during the day since the mask limits daily activities and may cause mid-face hypoplasia if introduced early in life. ${ }^{4}$

Children with CCHS present with respiratory, cardiovascular and ophthalmologic symptoms consistent with autonomic nervous system dysfunction. Ophthalmologic defects manifest as increased dilation (mydriasis) and decreased contraction (miosis) of the pupil to light stimuli. Patients may also present with lifethreatening brady-arrhythmias and asystoles which require a cardiac pacemaker implantation. Management is through a multidisciplinary approach both in hospital and community levels. In the hospitals, patients need coordinated care from respiratory, cardiac, radiology, surgery, genetics, and psychosocial counseling services. ${ }^{8}$ Regular evaluation needs to be done from diagnosis onward with the aim of optimizing outcome. Patients without Hirschsprung's disease may have symptoms of lower esophageal dysfunction and intestinal dysmotility resulting to constipation and abnormal esophageal motility. Screening for neural crest tumors is advised in children with 20/2820/33 genotypes in addition to all children with NPARMs. ${ }^{10}$ Home care plays a crucial role in the well-being of patients. They need to have a follow-up program upon discharge from the hospital, access to emergency care, home support nurses, and patient support groups. ${ }^{8}$

Long-term survival of patients with CCHS has significantly improved. It is important for clinicians to recognize congenital central hypoventilation syndrome in order to make an early diagnosis and prevent complications. Furthermore, confirmation of the diagnosis by PHOX2B gene analysis aids in genetic counseling and planning of optimal care of patients.

\section{Conclusion}

The case of J.D. contributes to the growing knowledge about CCHS and Haddad Syndrome. Had a correct diagnosis been made earlier, the severe infection could have been prevented with shorter hospital stay, and J.D. could have been sent home safely on assisted ventilation. It must be noted that an early diagnosis and confirmation by genetic testing is vital for the proper management of affected patients and prevention of complications. Despite the recent advances in the understanding and management of the disease, more genetic studies and genotype-phenotype correlation are needed to come up with recommendations which will aid in the diagnosis and management of patients affected with genetic disorders.

\section{Statement of Authorship}

All authors have approved the final version submitted.

\section{Author Disclosure}

All the authors declared no conflicts of interest.

\section{Funding Source}

None.

\section{References}

1. Tsoutsinos A, Karanasios E, Chatzis AC. Haddad Syndrome. Hellenic J Cardiol. 2016; 57(1):45-7.

2. Kaymacki A, Narter F, Yazar AS, Yilmaz MS. Congenital central hypoventilation syndrome with Hirschsprung's disease due to PHOX2B gene mutation in a Turkish infant. Turk J Pediatr. 2012; 54(5):519-22.

3. Chen ML, Keens TG. Congenital central hypoventilation syndrome: not just another rare disorder. Paediatr Respir Rev. 2004; 5(3):182-9.

4. Ljubic K, Fister Jr I, Fister I. Congenital central hypoventilation syndrome: a comprehensive review and future challenges. Journal of Respiratory Medicine. 2014; Article ID 856149.

5. Amiel J, Laudier B, Attie-Bitach T, et al. Polyalanine expansion and frameshift mutations of the paired-like homeobox gene PHOX2B in congenital central hypoventilation syndrome. Nat Genet. 2003; 33(4):459-61.

6. Weese-Mayer DE, Berry-Kravis EM, Ceccherini I, Keens TG, Loghmanee DA, Trang H. ATS Congenital Central Hypoventilation Syndrome Subcommittee. An official ATS clinical policy statement: Congenital central hypoventilation syndrome: genetic basis, diagnosis, and management. Am J Respir Crit Care Med. 2010; 181(6):626-44.

7. Trochet D, Hong SJ, Lim JK, et al. Molecular consequences of PHOX2B missense, frameshift and alanine expansion mutations leading to autonomic dysfunction. Hum Mol Genet. 2005; 14(23):3697-708.

8. Trang H, Brunet JF, Rohrer H, et al. European Central Hypoventilation Syndrome Consortium. Proceedings of the fourth international 
conference on central hypoventilation. Orphanet J Rare Dis. 2014; 9:194. doi: 10.1186/s13023-014-0194-5.

9. Preutthipan A, Kuptanon T, Kamalaporn H, Leejakpai A, Nugboon $\mathrm{M}$, Wattanasirichaigoon D. Using non-invasive bi-level positive airway pressure ventilator via tracheostomy in children with congenital central hypoventilation syndrome: two case reports. J Med Case Rep. 2015; 25(9):149. doi: 10.1186/s13256-015-0631-7.
10. Weese-Mayer DE, Rand CM, Zhou A, Carroll MS, Hunt CE. Congenital central hypoventilation syndrome: a bedside-to-bench success story for advancing early diagnosis and treatment and improved survival and quality of life. Pediatr Res. 2017; 81(1-2):192-201. doi: 10.1038/pr.2016.196.

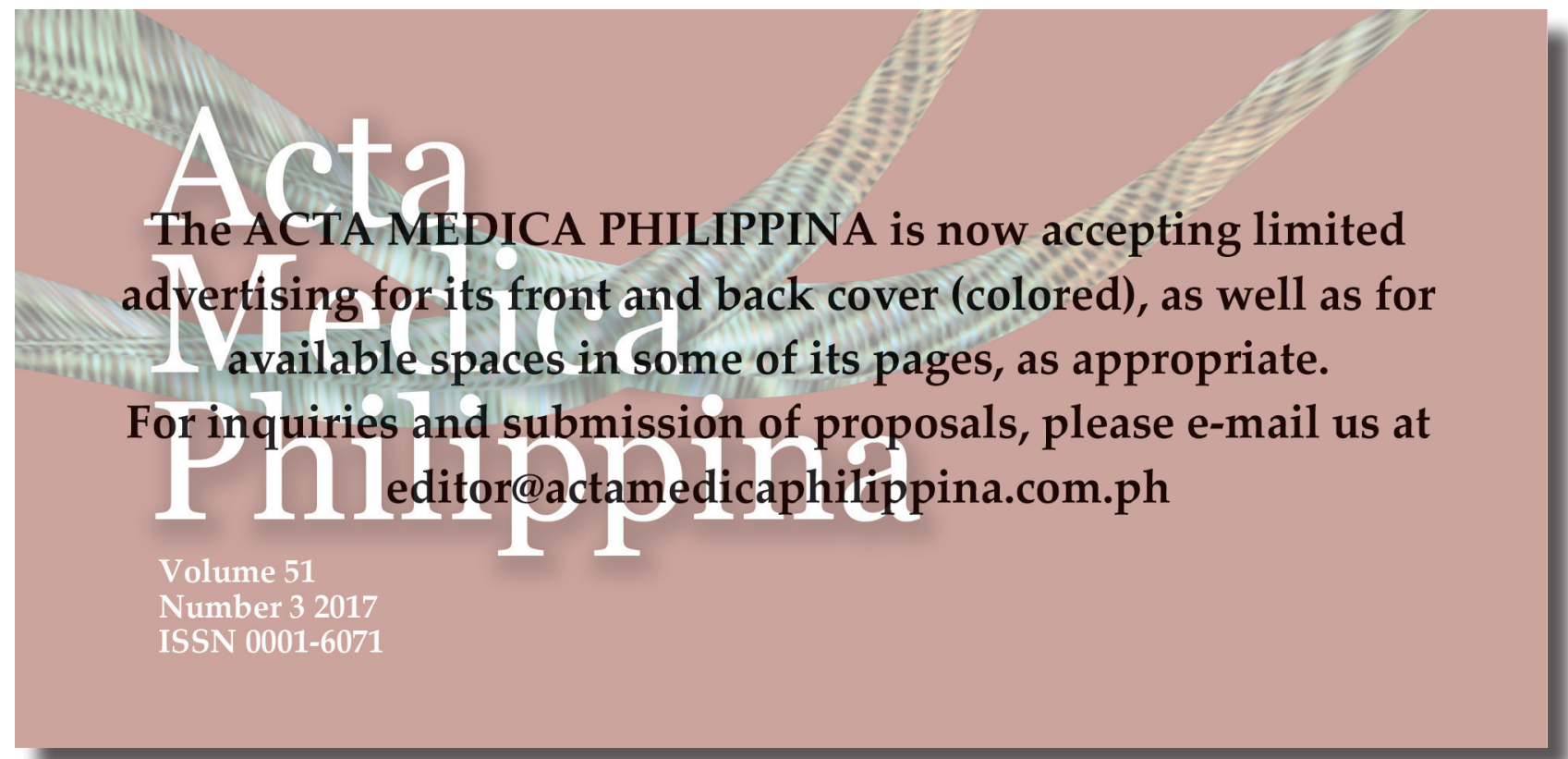

